\title{
Editorial: Introduction to Symposium on Ethics and Humanitarian Healthcare Policy and Practice
}

\author{
Matthew R. Hunt*, School of Physical and Occupational Therapy and Biomedical \\ Ethics Unit, McGill University \\ Lisa Schwartz, Department of Clinical Epidemiology and Biostatistics and \\ Centre for Health Economics \& Policy Analysis, McMaster University
}

*Corresponding author. Matthew Hunt, PhD, School of Physical and Occupational Therapy, McGill University, Hosmer House, Room 2053630 Promenade Sir William Osler, Montréal, Quebec, Canada, H3G 1Y5. Tel: +514-398-4400 (ext. 00289), Email: matthew.hunt@mcgill.ca

Humanitarian health assistance raises a range of ethical issues - and it does so at multiple levels. Whether the lens is focused close to consider practices and relationships within a disaster response project or dialed out to consider the role of nongovernmental organizations (NGOs) as international political actors, there is no shortage of ethical questions that merit careful analysis.

Five articles are included in this issue of Public Health Ethics looking at some of these issues. The authors of these papers have adjusted the focus of their analysis to bring into relief ethical questions at multiple levels of inquiry and from different perspectives. The contribution of bioethics researchers to examining ethical issues in humanitarian health ethics has so far been quite limited, but is growing quickly. Elaboration of normative guidance for policy setting and practice is a promising development (Hurst et al., 2009; Sheather and Shah, 2011). As well as exploring important ethical questions related to humanitarian health assistance, the diversity of themes presented in this issue is also suggestive of the potential scope of continued and expanded interdisciplinary analysis and discussion.

The papers collected here were originally presented in the context of a symposium held 26 and 27 November 2010, at McMaster University in Hamilton, Ontario, Canada, entitled 'Points of intersection: ethics, rights, health care \& public health in humanitarian health work'. The symposium brought together an interdisciplinary group of researchers, practitioners, students and representatives of aid organizations to examine how issues of ethics and rights related to practice and policy for humanitarian NGOs and their partners. The event was co-sponsored by the Center for Health Economics and Policy Analysis, McMaster University, the Center for Research on Ethics, University of
Montreal and the Center for the Study of Global Ethics, University of Birmingham and funded by the Canadian Institutes of Health Research.

Chung's contribution to the symposium is the widest in scope. She argues for the adoption of a more comprehensive understanding of the role and agency of humanitarian NGOs and articulates conceptual grounding to support such an approach. In advancing this thesis, she develops a critique of a narrowly conceived humanitarianism that does not acknowledge the politically entwined nature of such action and the inherent implications for social justice. Sounding a call for further debate, Chung illustrates potential implications in relation to pre-existing gender inequalities in societies where humanitarian health assistance is deployed, as well as gender considerations associated with the structures and implementation of aid projects. This article raises important questions regarding the nature of humanitarian NGOs as actors in the international sphere and in countries where programs are implemented, including how to understand the role of NGOs in relation to issues of inequality and social justice.

As an experienced humanitarian worker himself, Calain has had opportunity to struggle through ethical tensions of humanitarianism first hand. In his paper, he critically explores the question 'how legitimate is medical humanitarian action?' and the rationales expressed to justify humanitarian practice. He examines the notion of legitimate involvement and what constitutes appropriate participation in humanitarian actions. Calain also confronts the problem of what humanitarian action does to those they aim to assist, how 'helpers' mould the identities of others to create the 'helped'. The analysis asks how and to what extent humanitarian ideologies create policies that empower or disempower 
those involved. Calain's paper is a serious demand for reflective discourse on the role and place of humanitarian intervention, and leaves us with the lingering questions 'if not us, then who? And if not this way, then how?'

Kapiriri's paper poses significant questions about the effects on health priority setting of external agents such as foreign aid organizations. At a recent global health conference, the disorienting effects of funding were characterized as having profound impact on how countries reliant on foreign aid manage health priorities. Kapiriri's insightful empirical research has revealed the degree to which this is the case and she asks significant questions about the ethics of such involvement-is it needed assistance or lingering colonialism? Kapiriri's paper provides a balanced response that acknowledges the interests and requirements of all parties: sensitive to the crucial need for governments to remain self-defining and responsive to their countries' health requirements, but also aware of the pressures foreign aid organizations are under to be accountable for the way the funds are utilized.

The article led by Schwartz is the upshot of a collective research program by a multidisciplinary team, including bioethics researchers Hunt and Schwartz, sociologist Sinding, Adelson a medical anthropologist, Elit and Redwood-Campbell who are both doctors with extensive experience in humanitarian practice and De Laat a visual anthropologist. The article reflects one of the more striking findings in a qualitative study formed of interviews with humanitarian healthcare professionals on the ethical challenges they struggled with during work in the field. The narratives of participants revealed that health professionals often felt conflicted over their feelings of a duty of care for individual patients and the organizational remits that focused on collectives. The tension between the clinical ethics training health professionals arrive with from their home countries and the realities in the field that seemed to call for a public health ethics orientation is explored in this article with the intention of suggesting new directions for ethical research.

The paper by Hunt, Schwartz and Elit also focuses on the everyday ethical dimensions of healthcare and public health practice in NGOs and field projects. This article is drawn from the research program described above and is based on the narratives of practitioners. The authors present findings related to participants' experiences and perceptions of various forms of ethics support and training. Different NGOs, indeed different projects or divisions within the same NGO, adopt divergent approaches to support and training related to ethics. In this article, predeparture, in-field and postreturn phases of humanitarian work are considered in relation to ethics training and support. Slim has argued that NGO managers and leaders have a responsibility to 'ensure that their organization has a moral ethos and that their staff have the ethical skills and good knowledge of precedent with which to make and justify their moral decisions' (Slim, 1997: 255). This article can help inform discussion as NGOs seek to act on these responsibilities.

A primary objective of the Points of Intersection symposium was to encourage further research in humanitarian healthcare ethics and identify priority lines of inquiry. The ensuing dialogue suggested that there are clear needs for further empirical and conceptual research related to ethics and humanitarian assistance. As reflected by the symposium papers, such research includes broader conceptual analyses of humanitarian health assistance and its normative underpinnings, as well as research examining practices at the field level. In addition, more could be done to engender the interface of ethics with policy generation, revision and field practice on topics such as resource allocation, and identification of 'ethical exit strategies' for ending projects in ways that are sensitive to social and cultural needs of host communities. Finally, a research agenda needs to be conceived that would provide practitioners with tools and models for ethics support, mechanisms for managing moral distress, and better access to and understanding of the perceptions of partners and beneficiaries.

The points of intersection between ethics and humanitarianism are areas of extraordinarily rich moral experience. Ethics of humanitarianism at the broadest level is not a new area of study; analysis of ethical aspects of humanitarian healthcare work as it is provided in the field is still relatively nascent. Both require further study to map the intersections between ethics and humanitarian action, to support those who provide assistance to populations affected by war, disaster or deprivation to do so knowledgably and respectfully, and to avoid additional harm.

\section{References}

Hurst, S. A., Mezger, N. and Mauron, A. (2009). Allocating resources in humanitarian medicine. Public Health Ethics, 2, 89-99.

Sheather, J. and Shah, T. (2011). Ethical dilemmas in medical humanitarian practice: cases for reflection from Médecins Sans Frontières. Journal of Medical Ethics, 37, 162-165.

Slim, H. (1997). Doing the right thing: relief agencies, moral dilemmas and moral responsibility in political emergencies and war. Disasters, 21, 244-257. 